\title{
A Second-Order Two-Scale FEM Analysis for Time-Fractional Diffusion Equation with Small Periodic Coefficients
}

\author{
Ziqiang Wang \\ College of Science \\ Guizhou Minzu University \\ Guiyang, China \\ e-mail: wangzq@1sec.cc.ac.cn
}

\author{
Junying Cao \\ College of Science \\ Guizhou Minzu University \\ Guiyang, China \\ e-mail: caojunying1000@126.com
}

\begin{abstract}
In this paper, a second-order two-scale method for timefractional diffusion equation with rough periodic coefficients is presented by means of construction way. Based on the secondorder two-scale asymptotic expansion, the homogenization solution and the cell functions are obtained. The second-order twoscale approximate solution is constructed by the cell functions and the homogenization solution. Numerical experiments are carried out to support the theoretical claims.
\end{abstract}

Keywords- Second-order two-scale method; time-fractional diffusion equation; homogenization method; composite materials

\section{INTRODUCTION}

Fractional partial differential equations (FPDEs) appear in many science and engineering applications, which may be defined in some different but closely related ways. Some practical engineering examples of this kind of problems can be found in viscoelastic materials [1], advection and dispersion of solutes in natural porous or fractured media [2], hydrology [3], and so on. Along with the popularity of composite materials, the research on the mechanical behavior of the structure which is made of composite materials becomes an active field. It is logically sound to use the classical finite element method to analyze the structures with meshing all the details of microstructures, but it is not a practical way because it requires huge number of degrees of freedom to capture the micro-scale behavior. Fortunately, so many methods have been developed in the past decades, such as heterogeneous multiscale method [4], multiscale finite element method [5], finite element algorithms based on two-scale asymptotic analysis [6-8] and so on.

In this paper, we consider the time-fractional diffusion equation(TFDE) with rough periodic coefficients obtained from the standard diffusion equation by replacing the firstorder time derivative with a fractional derivative of order $\alpha$,with $0<\alpha \leq 1$.Time-fractional diffusion or wave equations are derived by considering continuous time random walk problems, which are in general non-Markovian processes.

However, published papers on the numerical solution of the TFDEs with rough periodic coefficients are very sparse. Here, we give a second-order two-scale (SOTS) computational method to TFDEs with rough periodic coefficents based on the ideas of [6-12].

$$
\begin{array}{r}
\text { Denote } \Omega \in R^{N}, \Sigma=\Omega \times[0, T], Y=[0,1]^{N} \text { and } y=\frac{x}{\varepsilon}, \\
x=\left(x_{1}, \cdots, x_{N}\right) \in \Omega . \text { Furthermore, } a_{i j}^{\varepsilon}(x)=a_{i j}(x / \varepsilon)
\end{array}
$$

$a_{i j}(y)$, which is Y-periodicity.

In the following parts, summations over repeated indices from 1 to $N$ are assumed. We hereafter denote by $C$ a generic positive constant which is independent of $\varepsilon$.

A. Constructing a second-order two-scale asymptotic expansion to TFDEs with rough periodic coefficents

We consider the following TFDE

$$
\begin{cases}D_{t}^{\alpha} u^{\varepsilon}(x, t)-\frac{\partial}{\partial x_{i}}\left(a_{i j}^{\varepsilon}(x) \frac{\partial u^{\varepsilon}(x, t)}{\partial x_{j}}\right)=f(x, t), & \text { in } \Sigma, \\ u^{\varepsilon}(x, t)=0,0 \leq t \leq T, & \text { on } \partial \Omega, \\ u^{\varepsilon}(x, 0)=0, & \text { in } \Omega .\end{cases}
$$

where $\alpha$ is the order of the time-fractional derivative. Here, we consider the case $0<\alpha \leq 1 \cdot D_{t}^{\alpha} u(x, t)$ in (1) is defined as the Caputo fractional derivatives of order $\alpha$, given by

$$
D_{t}^{\alpha} u^{\varepsilon}(x, t)=\frac{1}{\Gamma(1-\alpha)} \int_{0}^{t} \frac{\partial u^{\varepsilon}(x, \tau)}{\partial \tau} \frac{d \tau}{(t-\tau)^{\alpha}}, 0<\alpha \leq 1,
$$

and $a_{i j}^{\varepsilon}(x)$ satisfy

$$
\Lambda_{1} \eta_{i} \eta_{i} \leq a_{i j}^{\varepsilon}(x) \eta_{i} \eta_{j} \leq \Lambda_{2} \eta_{i} \eta_{i}, \forall \eta_{i} \in R^{N},
$$

where $\Lambda_{1}, \Lambda_{2}>0$.

Similar to [6-8], we construct the following SOTS's asymptotic expansion:

$$
u^{(2)}(x, t)=u^{0}(x, t)+\varepsilon P_{i}\left(\frac{x}{\varepsilon}\right) \frac{\partial u^{0}(x, t)}{\partial x_{i}}+\varepsilon^{2} Q_{i j}\left(\frac{x}{\varepsilon}\right) \frac{\partial^{2} u^{0}(x, t)}{\partial x_{i} \partial x_{j}},
$$

where $P_{i}\left(\frac{x}{\varepsilon}\right), Q_{i j}\left(\frac{x}{\varepsilon}\right), i, j=1,2, \cdots, N$. are defined as following:

(i) The local function $P_{k}(y)$ is Y-periodicity and defined on the reference cell $\mathrm{Y}$ as follow

$$
-\frac{\partial}{\partial y_{i}}\left(a_{i j}(y) \frac{\partial P_{k}(y)}{\partial y_{j}}\right)=\frac{\partial a_{i k}(y)}{\partial y_{i}}, \text { in } Y \text {. }
$$

(ii) The homogenization constants $a_{i j}^{0}$ are defined by

$$
a_{i j}^{0}=\int_{Y}\left(a_{i j}(y)+a_{i k}(y) \frac{\partial P_{j}(y)}{\partial y_{k}}\right) d y .
$$

(iii) The homogenization equation is defined by 


$$
\begin{cases}D_{t}^{\alpha} u^{0}(x, t)-\frac{\partial}{\partial x_{i}}\left(a_{i j}^{0} \frac{\partial u^{0}(x, t)}{\partial x_{j}}\right)=f(x, t), & \text { in } \Sigma, \\ u^{0}(x, t)=0,0 \leq t \leq T, & \text { on } \partial \Omega, \\ u^{0}(x, 0)=0, & \text { in } \Omega .\end{cases}
$$

(iv) The local function $Q_{i j}(y)$ is Y-periodicity and defined on the reference cell $\mathrm{Y}$ as follow

$$
\begin{aligned}
-\frac{\partial}{\partial y_{i}}\left(a_{i j}(y) \frac{\partial Q_{k l}(y)}{\partial y_{j}}\right)= & \frac{\partial}{\partial y_{i}}\left(a_{i l}(y) P_{k}(y)\right)+a_{l k}(y) \frac{\partial P_{k}(y)}{\partial y_{k}} \\
& +a_{k l}(y)-a_{k l}^{0}, \text { in } Y .
\end{aligned}
$$

\section{B. Approximation of SOTS's solution in pointwise sense}

In this section, we analyse the approximation of SOTS's solution. Let us introduce

$$
\delta^{(1)}(x, t)=u^{\varepsilon}(x, t)-u^{(1)}(x, t),
$$

and

$$
\delta^{(2)}(x, t)=u^{\varepsilon}(x, t)-u^{(2)}(x, t)
$$

where

$$
u^{(1)}(x, t)=u^{0}(x, t)+\varepsilon P_{i}\left(\frac{x}{\varepsilon}\right) \frac{\partial u^{0}(x, t)}{\partial x_{i}},
$$

and $u^{(2)}(x, t)$ is defined in (3).

Let us calculate $L^{\varepsilon}\left(\delta^{(1)}(x, t)\right)$ and $L^{\varepsilon}\left(\delta^{(2)}(x, t)\right)$ where $L^{\varepsilon}$ is defined as follows

$$
L^{\varepsilon}(\psi(x, t))=D_{t}^{\alpha} \psi(x, t)-\frac{\partial}{\partial x_{i}}\left(a_{i j}^{\varepsilon}(x) \frac{\partial \psi(x, t)}{\partial x_{j}}\right), \forall \psi(x, t) .
$$

Based on (4), (6), (7), (8) and taking into account that, $\frac{\partial}{\partial x_{i}} \rightarrow \frac{\partial}{\partial x_{i}}+\frac{1}{\varepsilon} \frac{\partial}{\partial y_{i}}$ one can formally obtain following residual to original equations in the pointwise sense

$$
\begin{aligned}
L^{\varepsilon}\left(\delta^{(1)}(x, t)\right) & =D_{t}^{\alpha} \delta^{(1)}(x, t)-\frac{\partial}{\partial x_{i}}\left(a_{i j}(y) \frac{\partial \delta^{(1)}(x, t)}{\partial x_{j}}\right) \\
& =f(x, t)-D_{t}^{\alpha}\left(u^{0}(x, t)+\varepsilon P_{i}(y) \frac{\partial u^{0}(x, t)}{\partial x_{i}}\right) \\
& +\frac{\partial}{\partial x_{i}}\left(a_{i j}(y) \frac{\partial}{\partial x_{j}}\left(u^{0}(x, t)+\varepsilon P_{k}(y) \frac{\partial u^{0}(x, t)}{\partial x_{k}}\right)\right) \\
& =D_{t}^{\alpha} u^{0}(x, t)-\frac{\partial}{\partial x_{i}}\left(a_{i j}^{0} \frac{\left.\partial u^{0}(x, t)\right)}{\partial x_{j}}\right) \\
& -D_{t}^{\alpha} u^{0}(x, t)-D_{t}^{\alpha}\left(\varepsilon P_{i}(y) \frac{\partial u^{0}(x, t)}{\partial x_{i}}\right) \\
& +\frac{\partial}{\partial x_{i}}\left(a_{i j}(y) \frac{\left.\partial u^{0}(x, t)\right)}{\partial x_{j}}\right) \\
& +\frac{\partial}{\partial x_{i}}\left(a_{i j}(y) \frac{\partial}{\partial x_{j}}\left(\varepsilon P_{k}(y) \frac{\partial u^{0}(x, t)}{\partial x_{k}}\right)\right)
\end{aligned}
$$

$$
\begin{aligned}
& =-\frac{\partial}{\partial x_{i}}\left(a_{i j}^{0} \frac{\left.\partial u^{0}(x, t)\right)}{\partial x_{j}}\right)-\varepsilon P_{i}(y) D_{t}^{\alpha}\left(\frac{\partial u^{0}(x, t)}{\partial x_{i}}\right) \\
& +\frac{\partial}{\partial x_{i}}\left(a_{i j}(y) \frac{\left.\partial u^{0}(x, t)\right)}{\partial x_{j}}\right) \\
& +\varepsilon \frac{\partial}{\partial x_{i}}\left(a_{i j}(y) \frac{\partial P_{k}(y)}{\partial x_{j}} \frac{\partial u^{0}(x, t)}{\partial x_{k}}\right) \\
& +\varepsilon \frac{\partial}{\partial x_{i}}\left(a_{i j}(y) P_{k}(y) \frac{\partial^{2} u^{0}(x, t)}{\partial x_{k} \partial x_{j}}\right) \\
& =-\frac{\partial}{\partial x_{i}}\left(a_{i j}^{0} \frac{\left.\partial u^{0}(x, t)\right)}{\partial x_{j}}\right)-\varepsilon P_{i}(y) D_{t}^{\alpha}\left(\frac{\partial u^{0}(x, t)}{\partial x_{i}}\right) \\
& +\varepsilon^{-1} \frac{\partial a_{i j}(y)}{\partial y_{i}} \frac{\partial u^{0}(x, t)}{\partial x_{j}}+a_{i j}(y) \frac{\left.\partial^{2} u^{0}(x, t)\right)}{\partial x_{j} \partial x_{i}} \\
& +\varepsilon^{-1} \frac{\partial}{\partial y_{i}}\left(a_{i j}(y) \frac{\partial P_{k}(y)}{\partial y_{j}}\right) \frac{\partial u^{0}(x, t)}{\partial x_{k}} \\
& +a_{i j}(y) \frac{\partial P_{k}(y)}{\partial y_{j}} \frac{\partial^{2} u^{0}(x, t)}{\partial x_{k} \partial x_{i}} \\
& +\frac{\partial}{\partial y_{i}}\left(a_{i j}(y) P_{k}(y)\right) \frac{\partial^{2} u^{0}(x, t)}{\partial x_{k} \partial x_{j}} \\
& +\varepsilon a_{i j}(y) P_{k}(y) \frac{\partial^{3} u^{0}(x, t)}{\partial x_{k} \partial x_{j} \partial x_{i}} \\
& =\left[\frac{\partial}{\partial y_{i}}\left(a_{i l}(y) P_{k}(y)\right)+a_{l k}(y) \frac{\partial P_{k}(y)}{\partial y_{k}}\right. \\
& \left.+a_{k l}(y)-a_{k l}^{0}\right] \frac{\partial^{2} u^{0}(x, t)}{\partial x_{k} \partial x_{l}} \\
& -\varepsilon N_{i}(y) D_{t}^{\alpha}\left(\frac{\partial u^{0}(x, t)}{\partial x_{i}}\right) \\
& +\varepsilon a_{i j}(y) P_{k}(y) \frac{\partial^{3} u^{0}(x, t)}{\partial x_{k} \partial x_{j} \partial x_{i}} .
\end{aligned}
$$

From (12), one obtains that the residual of FOTS's solution is $O(1)$ in pointwise sense with respect to $\varepsilon$, especially while the periodic $\varepsilon$ is a constant, does not tend to 0 even though it is small. This is not acceptable for the actual engineering calculation. That is why SOTS's solution must be considered. Next, we will give the pointwise approximation of SOTS's solution.

Similar to (12), a simple calculation shows that 


$$
\begin{aligned}
& L^{\varepsilon}\left(\delta^{(2)}(x, t)\right)=D_{t}^{\alpha} \delta^{(2)}(x, t)-\frac{\partial}{\partial x_{i}}\left(a_{i j}(y) \frac{\partial \delta^{(2)}(x, t)}{\partial x_{j}}\right) \\
& =f(x, t)-D_{t}^{\alpha}\left(u^{0}(x, t)+P_{i}(y) \frac{\partial u^{0}(x, t)}{\partial x_{i}}+Q_{i j}(y) \frac{\partial^{2} u^{0}(x, t)}{\partial x_{i} \partial x_{j}}\right) \\
& +\frac{\partial}{\partial x_{i}}\left(a_{i j}(y) \frac{\partial}{\partial x_{j}}\left(u^{0}(x, t)+P_{i}(y) \frac{\partial u^{0}(x, t)}{\partial x_{i}}+Q_{i j}(y) \frac{\partial^{2} u^{0}(x, t)}{\partial x_{i} \partial x_{j}}\right)\right. \\
& =D_{t}^{\alpha} u^{0}(x, t)-a_{i j}^{0} \frac{\partial^{2} u^{0}(x, t)}{\partial x_{j} \partial x_{i}}-D_{t}^{\alpha} u^{0}(x, t)-\varepsilon P_{i}(y) \frac{\partial D_{t}^{\alpha} u^{0}(x, t)}{\partial x_{i}} \\
& -\varepsilon Q_{i j}(y) \frac{\partial^{2} D_{t}^{\alpha} u^{0}(x, t)}{\partial x_{i} \partial x_{j}}+\varepsilon^{-1} \frac{\partial a_{i j}(y)}{\partial y_{i}} \frac{\partial u^{0}(x, t)}{\partial x_{j}}+a_{i j}(y) \frac{\partial^{2} u^{0}(x, t)}{\partial x_{i} \partial x_{j}} \\
& +\varepsilon^{-1} \frac{\partial}{\partial y_{i}}\left(a_{i j}(y) \frac{\partial}{\partial y_{j}} P_{k}(y)\right) \frac{\partial u^{0}(x, t)}{\partial x_{k}} \\
& +\frac{\partial}{\partial y_{i}}\left(a_{i j}(y) P_{k}(y)\right) \frac{\partial^{2} u^{0}(x, t)}{\partial x_{j} \partial x_{k}}+\frac{\partial}{\partial y_{i}}\left(a_{i j}(y) \frac{\partial}{\partial y_{j}} Q_{k l}(y)\right) \frac{\partial^{2} u^{0}(x, t)}{\partial x_{k} \partial x_{l}} \\
& +\varepsilon \frac{\partial}{\partial y_{i}}\left(a_{i j}(y) Q_{k l}(y)\right) \frac{\partial^{3} u^{0}(x, t)}{\partial x_{j} \partial x_{k} \partial x_{l}}+\varepsilon^{2} a_{i j}(y) Q_{k l}(y) \frac{\partial^{4} u^{0}(x, t)}{\partial x_{i} \partial x_{j} \partial x_{k} \partial x_{l}} \\
& =-\varepsilon N_{i}(y) \frac{\partial D_{t}^{\alpha} u^{0}(x, t)}{\partial x_{i}}-\varepsilon Q_{i j}(y) \frac{\partial^{2} D_{t}^{\alpha} u^{0}(x, t)}{\partial x_{i} \partial x_{j}} \\
& +\varepsilon \frac{\partial}{\partial y_{i}}\left(a_{i j}(y) Q_{k l}(y)\right) \frac{\partial^{3} u^{0}(x, t)}{\partial x_{j} \partial x_{k} \partial x_{l}}+\varepsilon^{2} a_{i j}(y) Q_{k l}(y) \frac{\partial^{4} u^{0}(x, t)}{\partial x_{i} \partial x_{j} \partial x_{k} \partial x_{l}} \\
& =\varepsilon F^{\varepsilon}(x, t)
\end{aligned}
$$

From (13), one can obtain the pointwise residual of SOTS' solution is $O(\varepsilon)$ to original problem (1) in $\Sigma$.

\section{Approximation theorem of SOTS's solution}

First we present a lemma which is Theorem 2.1 in [11], and we hereafter denote by $C$ a generic constant.

Lemma 1.1: For all $0<\alpha<1$ and $f(x, t) \in L^{2}(\Sigma)$, problem (1) is well-posed. Furthermore, if $u^{\varepsilon}(x, t)$ is the solution of (1), then it holds

$$
\left\|u^{\varepsilon}(x, t)\right\|_{B^{\frac{\alpha}{2}(\Sigma)}} \leq C\|f(x, t)\|_{0, \Sigma},
$$

where

$$
\|u\|_{B^{\frac{\alpha}{2}}(\Sigma)}=\left(\|u\|_{H^{\alpha / 2}\left((0, T): L^{2}(\Omega)\right)}^{2}+\|u\|_{H^{\alpha / 2}\left((0, T) H_{0}^{1}(\Omega)\right)}^{2}\right)^{1 / 2}, \forall u \in B^{\frac{\alpha}{2}}(\Sigma),
$$

and $\Sigma=\Omega \times(0, T)$, the other denotes are the same as [11].

The approximation analysis of SOTS's solution is given by the following result.

Theorem 1.1: Let $f(x, t) \in L^{2}(\Sigma), N_{k}(y), N_{k l}(y) \in W^{1, \infty}(Y)$, $k, l=1, \cdots, N$ and $u^{0}(x, t) \in H^{\frac{\alpha}{2}}\left((0, T), H_{0}^{3}(\Omega)\right)$.Then the SOTS's solution $u^{(2)}(x, t)$ satisfies following inequality

$$
\left\|u^{\varepsilon}(x, t)-u^{(2)}(x, t)\right\|_{B^{\frac{\alpha}{2}(\Sigma)}} \leq C \varepsilon^{1 / 2} .
$$

Proof: Based on (1) and (6), we have

$$
u^{\varepsilon}(x, 0)-u^{(2)}(x, 0)=0, \forall x \in \Omega,
$$

and

$$
\begin{aligned}
& u^{\varepsilon}(x, t)-u^{(2)}(x, t) \\
& =-\varepsilon N_{i}(y) \frac{\partial u^{0}(x, t)}{\partial x_{i}}-\varepsilon^{2} N_{i j}(y) \frac{\partial^{2} u^{0}(x, t)}{\partial x_{i} \partial x_{j}} \\
& \square-\varepsilon H^{\varepsilon}(x, t) .
\end{aligned}
$$

Denote $\theta^{\varepsilon}(x, t)=m^{\varepsilon}(x) H^{\varepsilon}(x, t)$, where $m^{\varepsilon}(x) \in C^{\infty}(\Omega)$ and satisfying

$\left\{\begin{array}{l}m^{\varepsilon}(x)=1, d(x, \partial \Omega) \leq \varepsilon, m^{\varepsilon}(x)=0, d(x, \partial \Omega) \geq 2 \varepsilon \\ 0 \leq m^{\varepsilon}(x) \leq 1,\left|\nabla m^{\varepsilon}(x)\right| \leq C \varepsilon^{-1}, \varepsilon \leq d(x, \partial \Omega) \leq 2 \varepsilon\end{array}\right.$

It is easy to see that

$$
\delta^{(2)}(x, t)+\varepsilon \theta^{\varepsilon}(x, t)=0, \text { on } \partial \Omega .
$$

Using the idea of Lemma 1.5 in chapter 1 of [12], we obtain

$$
\left\|\theta^{\varepsilon}(x, t)\right\|_{B^{\frac{\alpha}{2}(\Sigma)}} \leq C \varepsilon^{-1 / 2} .
$$

In virtue of (6) and (16), we have

$$
\delta^{(2)}(x, 0)+\varepsilon \theta^{\varepsilon}(x, 0)=0, \forall x \in \Omega \text {. }
$$

In virtue of (13), (19),(21) and Lemma1.1, we obtain

$$
\begin{aligned}
& \left\|\delta^{(2)}(x, t)+\varepsilon \theta^{\varepsilon}(x, t)\right\|_{B^{\frac{\alpha}{2}}(\Sigma)} \\
& \leq\left\|\varepsilon F^{\varepsilon}(x, t)\right\|_{L^{2}(\Sigma)}+\left\|\varepsilon \nabla \theta^{\varepsilon}(x, t)\right\|_{L^{2}(\Sigma)} \\
& \leq C \varepsilon+C \varepsilon\left\|\theta^{\varepsilon}(x, t)\right\|_{B^{\frac{\alpha}{2}}(\Sigma)}
\end{aligned}
$$

Using the triangle inequation and (20), we have

$$
\begin{aligned}
& \left\|\delta^{(2)}(x, t)\right\|_{B^{\frac{\alpha}{2}}(\Sigma)} \\
& \leq\left\|\delta^{(2)}(x, t)+\varepsilon \theta^{\varepsilon}(x, t)\right\|_{B^{\frac{\alpha}{2}}(\Sigma)}+\left\|\varepsilon \theta^{\varepsilon}(x, t)\right\|_{B^{\frac{\alpha}{2}}(\Sigma)} \\
& \leq C\left(\varepsilon+\varepsilon^{1 / 2}\right) .
\end{aligned}
$$

The proof is then complete.

\section{NUMERICAL SCHEME USING FEM BASED ON SECOND ORDER TWO SCALE ASYMPTOTIC EXPANSION}

In this section, we present the numerical scheme for solving diffusion equation rough periodic coefficients by using SOTS's asymptotic expansion. The algorithm procedure of SOTS's computation is following:

(i) Solve the equation (4) using FE numerical method on cell Y to obtain $P_{k}^{\left(h_{0}\right)}(y)$. And then evaluate the homogenized constitutive coefficients $a_{i j}^{0\left(h_{0}\right)}$ in formula (5) with $P_{k}^{\left(h_{0}\right)}(y)$ instead of $P_{k}(y), k=1, \cdots, N$.

(ii) Solve the equation (6) using FE numerical method in $\Omega$ to obtain $u^{0(h)}$. And then evaluate its partial derivatives $\frac{\partial^{2} u^{0(h)}}{\partial x_{k} \partial x_{l}}, k, l=1, \cdots, N$, using high order difference quotient in [7].

(iii) Solve the equation (7) using FE numerical method on cell $\mathrm{Y}$ with the same mesh in (ii) to obtain $Q_{k l}^{\left(h_{0}\right)}(y), k, l=1, \cdots, N$. 
(iv) Substituting $P_{k}^{\left(h_{0}\right)}(y), u^{0(h)}$ and $Q_{k l}^{\left(h_{0}\right)}(y), k, l=1, \cdots, N$. into (3), we obtain the SOTS's computational method to $u^{\varepsilon}$.

\section{NUMERICAL EXAMPLES}

In order to show the effectiveness of the SOTS's method we give some numerical results for TFDE with rough periodic coefficients. The numbers of elements and nodes are 57088 and 28865, respectively, for computing the original problem (1), where $f=10, \alpha=0.9, \Omega=[0,1]^{2}, \varepsilon=1 / 8$, $Y=[0,1]^{2}$, which are shown in Fig. 1 , and the constants of equation are:

$$
a_{i j}(y)= \begin{cases}\delta_{i j}, & \text { in } Y_{1}, \\ 0.01 \delta_{i j}, & \text { in } Y_{2} .\end{cases}
$$

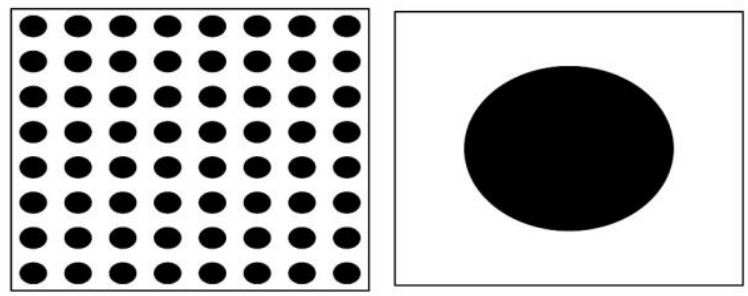

Fig. 1. The model of 2-D composite domain $\Omega=[0,1]^{2}$ (left), the reference cell $Y=[0,1]^{2}$ (right).

The results of the homogenization constants, which are computed by SOTS's methods, are

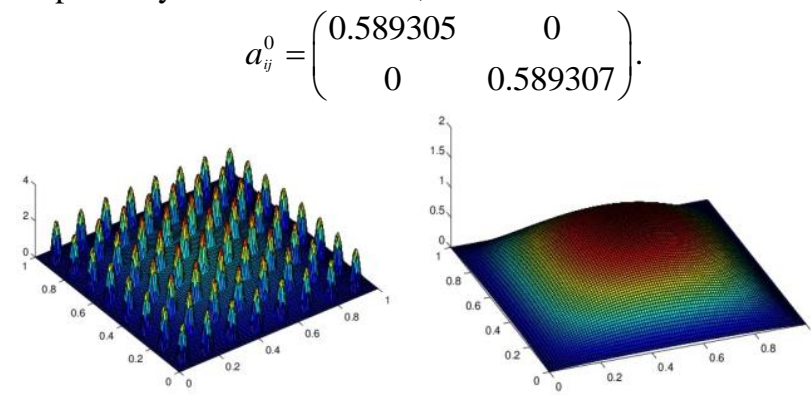

Fig. 2. The FEM solution of $u^{i}(x, 0.4)$ in refined mesh(left),

the homogenization solution $u^{0}(x, 0.4)$ (right).
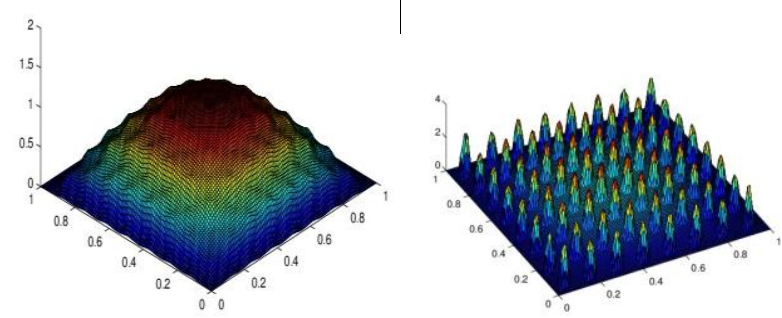

Fig. 3. The FOTS's solution of $u^{(1)}(x, 0.4)$ (left),

SOTS's solution $u^{(2)}(x, 0.4)$ (right).

From Fig. 2-3, it is easy to see that: The first-order twoscale(FOTS) approximate solution $u^{(1)}(x, t)$ is certain approximation to $u^{\varepsilon}(x, t)$, and can only capture partly of the microscopic behaviors caused from micro-structures. The SOTS's approximate solution $u^{(2)}(x, t)$ is better approximation to $u^{\varepsilon}(x, t)$ and can capture more precisely the microscopic 2-D behaviors caused from 2-D microstructures than $u^{(1)}(x, t)$.

\section{ACKNOWLEDGMENT}

This work was partially supported by the Foundation of Guizhou Science and Technology Department (Grant No.[2013]2144), Guizhou Key Laboratory of Pattern Recognition and Intelligent System Construction Project(Grant No. [2009]4002) and also supported by 2013 Key project of scientific research project of Guizhou Minzu University.

\section{REFERENCES}

[1] R.L. Bagley and R.A. Calico, "Fractional order state equations for the control of viscoelastically damped structures", Journal of Guidance, Control, and Dynamics, vol 4,1991,pp.304-311, doi:10.2514/3.20641.

[2] D.A. Benson, S.W. Wheatcraft, and M.M. Meerschaert. "Application of a fractional advection-dispersion equation". Water Resour. Res. vol.36,Feb.2000,pp.1403-1412, doi:10.1029/2000WR900031.

[3] R. Schumer, D.A. Benson, M.M. Meerschaert, and B. Baeumer "Multiscaling fractional advection-dispersion equations and their solutions". Water Resour. Res., vol.39,Jan.2003,pp.1022 - 1032, doi:10.1029/2001WR001229.

[4] E. Weinan, P. B. Ming and P. W. Zhang, "Analysis of the heterogeneous multiscale method for elliptic homogenization problems," J. Amer. Math. Soc., vol.18,Sep.2005,pp.121-156, doi:10.1090/S0894-0347-04-00469-2.

[5] T. Y. Hou and X. H. Wu, "A multiscale finite element method for elliptic problems in composite materials and porous media," J. Comput. Phys., vol.134,Jun.1997, pp. 169-189, doi:10.1006/ jcph.1997.5682.

[6] J. Z. Cui,T. M. Shih and Y. L. Wang, "The two-scale analysis method for bodies with small periodic configurations," Struct. Eng. Mech. vol.7,no.6,pp.601-614, 1999

[7] J. Z. Cui and H. Y. Yang, "A dual coupled method for boundary value problems of PDE with coefficients of small periodic," J. Comput. Math., vol. 14,pp.159-174,1996.

[8] Z.Q. Wang and J.Y. Cao "Second Order Two-scale Method for Composite Plate with 3-D Periodic Configuration under Condition of Coupled Thermoelasticity," Advanced Materials Research, vol.898,2014,pp.7-10, doi:10.4028/www.scientific.net/AMR.898.7.

[9] J.Y. Cao, C.J. Xu and Z.Q. Wang, "A high order finite difference/ spectrial approximations to the time fractional diffusion equations," Advanced Materials Research, vol.875-877,2014,pp.781-785, doi:10.4028/www.scientific.net/AMR.875-877.781.

[10] Y.M Lin and C.J Xu, "Finite difference/spectral approximations for the time-fractional diffusion equation," J. Comput. Phys, vol.225, Aug.2007,pp. 1533-1552, doi:10.1016/j.jcp.2007.02.001.

[11] X.J. Li and C.J Xu, "A space-time spectral method for the time fractional diffusion equation," Siam J. Numer. Anal., vol.47, 2009,pp. 2108-2131, doi:10.1137/080718942.

[12] O. A. Oleinik , A. S. Shamaev and G. A. Yosifian, Mathematical problems in elasticity and homogenization. Amsterdam:North Holland, 1992 